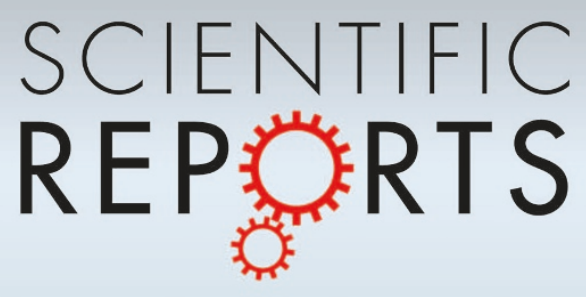

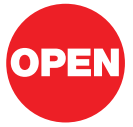

SUBJECT AREAS:

MIDBRAIN

PERCEPTION

NEUROPHYSIOLOGY

BIOMEDICAL ENGINEERING

Received

21 November 2012

Accepted

25 February 2013

Published

18 March 2013

Correspondence and requests for materials should be addressed to H.H.L. (hlim@umn.edu)

\section{Frequency representation within the human brain: Stability versus plasticity}

\author{
Hubert H. Lim ${ }^{1,2}$, Minoo Lenarz ${ }^{3}$, Gert Joseph ${ }^{4}$ \& Thomas Lenarz ${ }^{4}$
}

'Department of Biomedical Engineering, University of Minnesota, Minneapolis, MN, USA, ${ }^{2}$ Institute for Translational Neuroscience, University of Minnesota, Minneapolis, MN, USA, ${ }^{3}$ Department of Otolaryngology, Berlin University - Charite, Berlin, Germany, ${ }^{4}$ Department of Otolaryngology, Hannover Medical University, Hannover, Germany.

A topographical representation for frequency has been identified throughout the auditory brain in animals but with limited evidence in humans. Using a midbrain implant, we identified an ordering of pitch percepts for electrical stimulation of sites across the human inferior colliculus (IC) that was consistent with the IC tonotopy shown in animals. Low pitches were perceived by the subject for stimulation of superficial IC sites while higher pitches were perceived for stimulation of deeper sites. Interestingly, this pitch ordering was not initially observed for stimulation across the IC, possibly due to central changes caused by prior hearing loss. Daily implant stimulation for about 4 months altered the pitch percepts from being predominantly low to exhibiting the expected ordering across the stimulated IC. A presumably normal tonotopic representation may have been maintained within the IC or accessible through IC stimulation that helped form this pitch ordering perceived in higher centers.

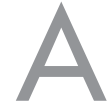

fundamental property of the brain is the representation of sensory receptors across spatially ordered neurons from the periphery up to the cortex. For example, haptic stimuli across different skin locations are topographically mapped within the spinal cord, brainstem, thalamus and cortex; and different sound frequencies are topographically mapped within the cochlea, brainstem, midbrain, thalamus and cortex. Remarkably, these sensory maps can be modified, even in adulthood, through altered or reinforced input (e.g., peripheral damage, altered stimulus environments, training or fear conditioning paradigms) ${ }^{1-5}$. Central plasticity occurs predominantly in thalamocortical pathways though changes have also been observed in lower subcortical nuclei ${ }^{3,4,6-11}$.

What remains puzzling is how the sensory brain can adapt to an altered or reinforced input without losing the ability to switch back to its previous coding state. In particular, it is possible to shift the sensitivity of central auditory neurons to specific frequencies through behavioral training, fear conditioning, or paired acousticneuromodulatory stimulation ${ }^{8,12-15}$. Over time shifted neurons can return back to their original state through passive listening. Even under conditions of peripheral damage caused by loud sounds, ototoxic drugs, or progressive hearing loss, frequency organization (i.e., tonotopy) in the auditory cortex can be compromised; yet through crude and uneven peripheral activation using pure tones or cochlear stimulation, tonotopy consistent with the original representation can be restored ${ }^{16-18}$. One possibility based on previous animal studies ${ }^{9,10,19,20}$ is that the auditory brain can maintain an original representation for frequency within brainstem and/or midbrain centers, while tonotopic reorganization occurs predominantly within thalamocortical pathways. In this way, the brain could achieve both stability and plasticity to changing conditions through appropriate ascending and descending coordination between the fixed and plastic representations ${ }^{8,10,19,21,22}$.

We implanted a deaf patient with an auditory prosthetic electrode array within the inferior colliculus (IC) in which sites were positioned within the central nucleus of the IC (ICC), the main ascending auditory nucleus within the midbrain. Numerous studies in animals have shown a low to high frequency representation along the dorsolateral to ventromedial gradient of the $\mathrm{ICC}^{23-33}$. However, there is still no functional evidence for a frequency organization across the human ICC. Therefore, we had a unique opportunity to investigate tonotopy in our ICimplanted patient. Electrode sites were aligned along the dorsomedial to ventrolateral axis of the ICC and we electrically stimulated each site to assess the different pitch percepts. We present data demonstrating a pitch ordering for stimulation across the human ICC consistent with the tonotopy observed in the previous animal studies mentioned above. Interestingly, this pitch ordering was not observed until after 4 months of daily stimulation with the midbrain implant. Stimulation across the ICC initially elicited predominantly low pitch percepts. The patient had severe high frequency hearing loss prior to midbrain stimulation that may have caused central neurons to code for predominantly low frequencies ${ }^{9,20}$. Daily implant stimulation for about 4 months was 
then able to alter the pitch percepts from being predominantly low to exhibiting the expected ordering across the stimulated ICC. Based on our data and previous animal findings $s^{9,10,19,20}$, it is possible that a presumably normal tonotopic representation may have been maintained within the ICC or accessible through ICC stimulation that helped form this ordered pitch representation that is perceived in higher centers.

\section{Results}

Tonotopy within the human ICC. We investigated tonotopy in an adult deaf patient implanted with an auditory prosthetic electrode array into the right IC with sites positioned across the ICC (Fig. 1; see Experimental Procedures for further patient details). Surprisingly, electrical stimulation of different sites across the hypothesized frequency axis of the ICC elicited predominantly low pitch percepts within the first 1.5 months of implant use (Fig. 2a). Stimulation of sites 1 to 6 correctly exhibited an increase in pitch, but stimulation of deeper sites elicited low pitches. As discussed in the next section, it is possible that the predominance of low pitch percepts may have been caused by tonotopic reorganization due to high frequency hearing loss the patient experienced before complete deafness and midbrain stimulation. However, after 10 months of daily implant use, a low to high pitch ordering was observed for the stimulated sites across the dorsolateral to ventromedial axis of the ICC consistent with the tonotopy identified in animals. Similar results were obtained using a pitch ranking procedure. Figure $2 b$
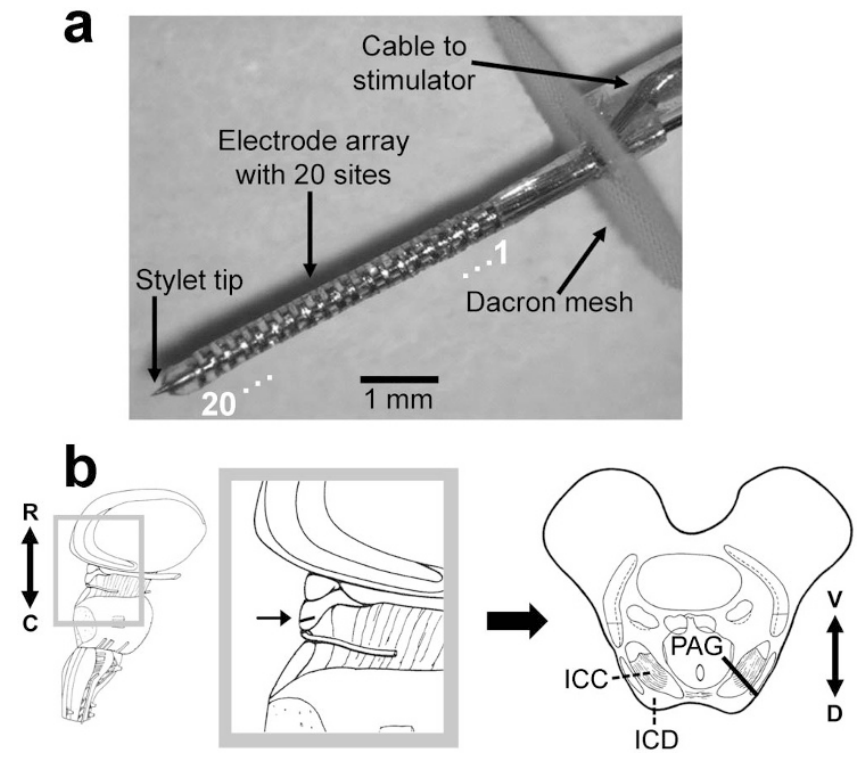

Figure $1 \mid$ Electrode array implantation across the right ICC. (a) The array consists of 20 platinum ring electrodes linearly spaced at $200 \mu \mathrm{m}$ along a silicone carrier. Each site has a thickness of $100 \mu \mathrm{m}$ and an area of $0.126 \mathrm{~mm}^{2}$. A stainless steel stylet through the center of the silicone array enables insertion into the brain and is removed after proper array placement. (b) Parasagittal (middle) and axial (right) sections show the location of the array (black line). An arrow in the parasagittal section points to the caudal-rostral location of the array and also corresponds to the location of the axial section shown to the right derived from CT and MRI images superimposed onto fixed human midbrain slices. The array spans the expected low to high frequency gradient from the superficial to deeper layers of the ICC (dorsal-caudal portion) based on animal studies. Right ICC stimulation elicited sounds perceived as coming from the left ear. The tip sites 12 to 20 were located in deeper non-auditory regions (e.g., periaqueductal gray, PAG) and were inactivated. Images in this figure were taken from previous publications ${ }^{39,43}$ and reprinted with permission from Society for Neuroscience and Wolters Kluwer Health. ICD: dorsal cortex of inferior colliculus, C: caudal, D: dorsal, R: rostral, V: ventral.

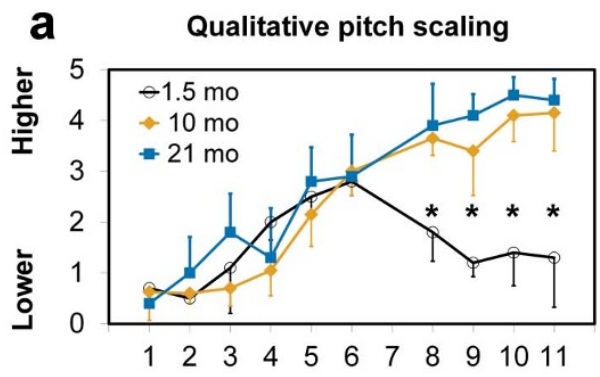

b 2-AFC pitch ranking

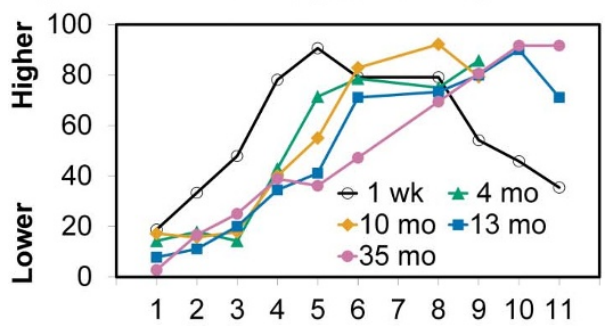

C Quantitative pitch scaling

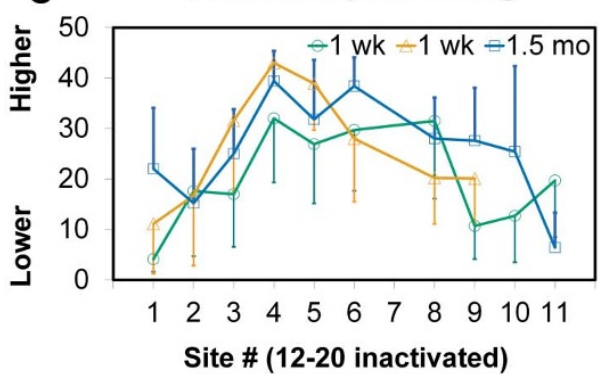

Figure $2 \mid$ Pitch ordering over time for three different tests. (a) Subject indicated a value from 0 to 5 for each stimulated site based on a pitch scale of familiar objects ( 0 : bass or boat horn, 2: man's voice, 3.5 : woman's voice, 5: bird chirping). Average and standard deviation across $n$ trials are plotted for each site (1.5 mo: $n=5,10 \mathrm{mo}: n=10,21 \mathrm{mo}: n=5)$. Asterisks denote significantly higher 10 - and 21 -month values than 1.5 -month values $(\mathrm{p}<0.006$, two-tailed ranked unequal variance $\mathrm{t}$-test). (b) Two-alternative forced choice (2-AFC) ranking method required the subject to indicate which site out of two sequentially stimulated sites had a higher pitch. All sites were then rank ordered based on how often each site had a higher pitch over all other sites (in percentage). Each site pair was compared $n$ times ( $1 \mathrm{wk}: n=12,4$ mo: $n=4,10 \mathrm{mo}: n=20,13 \mathrm{mo}: n=10,35 \mathrm{mo}$ : $n=4$ ). (c) Subject indicated a number from 0 to 50 (low to high pitch) for each site. Average and standard deviation across $n$ trials is plotted for each site (two sessions for 1 wk: $n=10$ each, 1.5 mo: $n=5$ ). For each test, all sites were stimulated in a random sequence and at a similar loudness level. Site 7 was shorted to a non-auditory site and was excluded.

shows that the expected pitch ordering was mostly present after 4 months of implant use. A third pitch method (Fig. 2c) also showed a similar pattern as in Fig. $2 \mathrm{a}$ and $2 \mathrm{~b}$ during the initial 1.5 -month period. Sites 4 through 6 usually had the highest pitch percepts while the deeper sites had unexpectedly lower pitch percepts. We did not use this third pitch method for later testing sessions due to its higher variability across trials compared to the other two methods.

Stable versus plastic frequency representation. Using two different pitch tests, we consistently observed a pitch ordering along the dorsolateral to ventromedial gradient of the ICC consistent with the tonotopy shown in animals. However, this supposedly normal frequency organization was not present until after 1.5-4 months of daily implant stimulation. Only low pitches rather than high pitches 
were perceived by the patient when stimulating the deeper sites of the array for that initial period. As shown in Fig. 3, we did not observe any systematic differences in activation levels across deeper versus shallower sites over time. Therefore, it is unlikely there was greater neural damage surrounding those deeper sites (i.e., presumably the higher frequency regions) caused by array implantation that would initially require higher current levels to activate functional neurons surrounding the more shallow sites (i.e., presumably the lower frequency regions) to elicit an auditory percept until recovery of the damaged neurons.

An alternative explanation is that our patient experienced tonotopic reorganization due to high frequency hearing loss (Fig. 4a), only hearing low frequencies below $1 \mathrm{kHz}$ in the left ear that predominantly projects to the right implanted ICC. The subject was already deaf in the right ear for about 10 years before experiencing significant hearing loss in the left ear. Based on animal studies, low frequency thalamocortical expansion would occur due to this high frequency hearing $\operatorname{loss}^{9}$, and thus subsequent stimulation across the ICC could elicit predominantly low pitch percepts that are coded within those higher auditory centers, consistent with our pitch results in Fig. 2. Surprisingly, non-tonotopic stimulation across the ICC for the first 10 months resulted in a systematic pitch ordering as expected from the tonotopy identified in animals. We describe the stimulation as non-tonotopic because different frequency components of the incoming sound were presented as electrical pulses to specific ICC sites that did not match the expected frequency ordering of the ICC. For example, the highest frequency sound components were presented to the middle ICC sites, which are supposedly middle frequency regions, and middle frequency components were presented to deeper sites, which are supposedly higher frequency regions (Fig. 4b). Therefore, it was not possible for the patient to associate and re-learn the expected pitch ordering based on the inputted sound and environmental cues to achieve the 4 - and 10-month pitch results shown in Fig. 2. If the changes in pitch percepts were truly driven by what was presented to the different ICC sites, then the pitch ordering should have converged towards the list shown for 0-4 months or 4-10 months in Fig. $4 \mathrm{~b}$ for the testing session at 4 months or 10 months, respectively, which was not the case. Instead, we propose that the expected frequency ordering may have been maintained within the ICC or at least accessed through ICC stimulation even though tonotopic reorganization occurred within higher perceptual centers during hearing loss. This is not to claim that changes in frequency coding did not occur within the ICC or even in lower

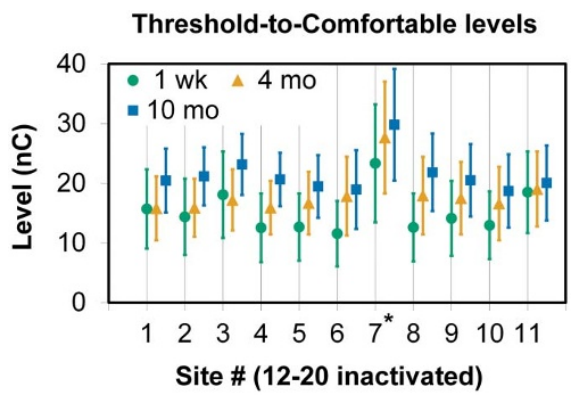

Figure 3 Activation levels over time. Threshold and upper comfortable levels were measured in terms of total charge per phase of the biphasic pulses presented on each site and plotted as a vertical line with a symbol at the midpoint. Site 7 was electrically shorted to a distant non-auditory site and required higher activation levels. Levels generally increased over time, but there were no systematic differences in the values or change in values for the deeper sites compared to the shallower sites. It would be expected that if there was greater damage and/or recovery of neurons surrounding the deeper sites (i.e., sites $8-11$ ), then activation levels should have been higher and/or levels should have changed differently over time compared to the shallower sites.

\section{a Audiogram before deafness onset}

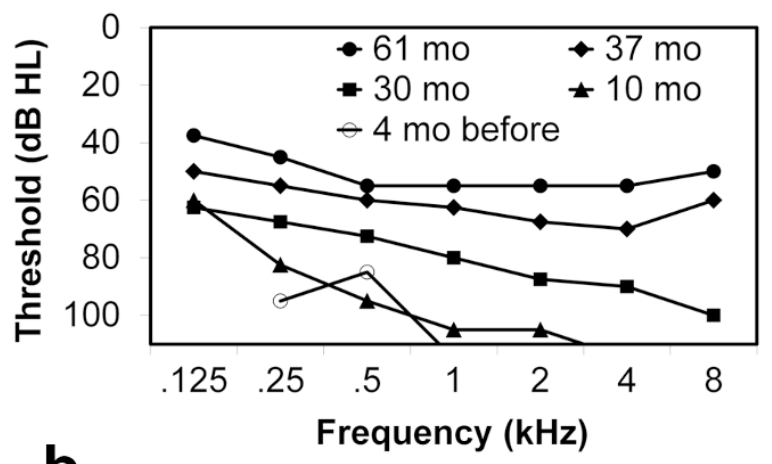

Implant processor frequency-to-electrode mapping

\begin{tabular}{|c|c|c|c|c|c|c|c|c|}
\hline \multicolumn{3}{|c|}{ 0-4 months } & \multicolumn{3}{|c|}{ 4-10 months } & \multicolumn{3}{|c|}{ 10-21 months } \\
\hline LowF & HighF & Site \# & LowF & HighF & Site \# & LowF & HighF & Site \# \\
\hline 188 & 313 & 11 & 188 & 438 & 1 & 188 & 313 & 1 \\
\hline 313 & 563 & 1 & 438 & 688 & 2 & 313 & 563 & 2 \\
\hline 563 & 813 & 2 & 688 & 1063 & 3 & 563 & 813 & 3 \\
\hline 813 & 1063 & 3 & 1063 & 1438 & 4 & 813 & 1063 & 4 \\
\hline 1063 & 1438 & 10 & 1438 & 2063 & 8 & 1063 & 1438 & 5 \\
\hline 1438 & 1938 & 9 & 2063 & 2938 & 9 & 1438 & 1938 & 6 \\
\hline 1938 & 2563 & 6 & 2938 & 4063 & 5 & 1938 & 2563 & 7 \\
\hline 2563 & 3438 & 8 & 4063 & 5688 & 7 & 2563 & 3438 & 8 \\
\hline 3438 & 4563 & 7 & 5688 & 7938 & 6 & 3438 & 4563 & 9 \\
\hline 4563 & 6063 & 5 & & & & 4563 & 6063 & 10 \\
\hline 6063 & 7938 & 4 & & & & 6063 & 7938 & 11 \\
\hline
\end{tabular}

Figure 4 | The expected pitch ordering produced by non-tonotopic activation of the right ICC. (a) Hearing thresholds for the left ear before deafness onset caused by acoustic neuroma removal surgery. Sounds entering the left ear project predominantly to the right ICC. (b) Different frequency components (between LowF to HighF in $\mathrm{Hz}$ ) of incoming sound are electrically presented on each site for daily implant use. Non-tonotopic activation across the right ICC (i.e., without a systematic ordering of sites that matches the tonotopy of the ICC shown in animal studies) during the first 10 months of stimulation produced the expected pitch ordering shown in Fig. 2 that was supposedly altered by the high frequency hearing loss shown in (a).

centers that then project to the ICC. Rather, we are just suggesting that non-tonotopic stimulation across the ICC for several months produced the expected pitch ordering perceived in higher centers because an original frequency (or pitch) representation was somehow maintained within the central auditory system that could be accessed by ICC stimulation.

\section{Discussion}

Tonotopy has been identified across the dorsolateral to ventromedial gradient of the ICC across mammalian species, including the mouse, rat, cat, guinea pig, gerbil, chinchilla, ferret, and monkey ${ }^{23-33}$. Considering that two dimensional anatomical laminae have been identified in the human ICC $^{34}$ and are consistent with the isofrequency laminae observed in animals, it was inferred that tonotopy also exists across the human ICC. We presented results demonstrating that electrical stimulation across the hypothesized tonotopic gradient of the human ICC does result in the expected pitch percepts in which low to high pitches were observed for superficial to deeper regions.

The tonotopic representation in the ICC appears to be quite robust to peripheral auditory damage. Several studies in adult animals have shown that peripheral cochlear damage caused by noise trauma or mechanical lesions induces frequency reorganization within the primary auditory cortex and the ventral division of the medial geniculate body, but limited frequency changes within the $\mathrm{ICC}^{9-11,19,20}$. In 
particular, neurons previously tuned to the frequencies of the damaged region (e.g., high frequencies) become retuned to the edge and/or remaining frequencies, resulting in an expanded representation for those frequencies (e.g., lower frequencies) within the thalamus and cortex. Based on those findings, it has been suggested that frequency plasticity may largely occur within the thalamocortical circuits, while the ICC and even the cochlear nucleus may provide tonotopic stability during brain plasticity, at least during peripheral hearing damage $e^{10,11,19,35}$. If an original frequency representation was maintained within the ICC, non-tonotopic stimulation across the ICC may still have activated and reinforced a sufficient number of frequency channels up to higher perceptual centers, possibly shifting or resetting the predominantly low pitch representation towards a pitch ordering consistent with the tonotopy of the stimulated ICC.

Further support for the dual existence of fixed and plastic neural representations for sound features is based on sound localization studies. In both humans and ferrets, it has been shown that subjects can relearn cues for a sound localization task when plugs are inserted into the ears over several weeks. Surprisingly, after the ear plugs are removed, the subjects can immediately return back to their original sound localization cues ${ }^{22,36}$, suggesting that the previous representation was maintained within the brain during development of the new representation. There is also direct physiological evidence based on eloquent studies in barn owls demonstrating the co-existence of the original and learned neural maps for sound localization cues that are coded within the external nucleus of the $\mathrm{IC}^{5,37}$. Therefore, the brain appears to be capable of maintaining a dual fixed and plastic representation for sound features, which may enable the sensory brain to experience plasticity while still maintaining stability.

\section{Methods}

Subject. A female subject was implanted with an auditory midbrain implant array (AMI; developed by Cochlear Limited, Australia) in the right IC with sites positioned within the ICC (Fig. 1) at the age of 42 in 2006. She had been diagnosed with neurofibromatosis type II, which is a genetic disease associated with peripheral nerve tumors, including bilateral acoustic neuromas. In 1986, she underwent surgery to remove an acoustic neuroma on the right side that led to deafness on that side. In 2001 , she had another acoustic neuroma removed on the left side that led to complete deafness. Due to the tumor, she experienced high frequency hearing loss in the left ear with rising low frequency thresholds up until complete deafness caused by the tumor removal surgery (Fig. 4a). Audiograms were measured using standard clinical procedures in Germany. For each frequency, the pure tone amplitude was increased until the subject indicated that a sound was perceived. This measurement was repeated several times until the responses were consistent across trials. The thresholds were measured using a calibrated headphone and clinical audiometric device (4-month data: Madsen Orbiter 922, Otometrics, Denmark; all other data: Beoton Beomat 2005, Ollmann, Germany).

The subject had a soft bilateral noise-like tinnitus. The subject did not notice any change in her tinnitus due to AMI implantation or stimulation except that the tinnitus could be masked by the electrically-induced sound sensations. Stimulation of only one side may not be sufficient to alter the bilateral tinnitus.

All procedures were conducted in accordance with ISO 14155 and the Good Clinical Practice guidelines. Medical Ethics Committee and Competent Authority written approvals through Hannover Medical University in Germany and according to national laws were obtained and the patients signed informed consent forms prior to AMI implantation and testing.

Array location. AMI implantation and array localization methods have been previously described ${ }^{38,39}$. Briefly, three-dimensional MRI brain slices were obtained for the subject prior to AMI implantation using a 1.5 Tesla magnet (Allegra, Siemens, Germany). Accurate MRI images of the midbrain could not be obtained after AMI implantation due to the distortion caused by the array and telemetry interface magnet. Instead, CT images (Light Speed VFX, GE Medical Systems, USA) were obtained for the subject 6 weeks after AMI implantation, which was sufficient time to avoid brain swelling caused by the surgery. The MRI data was then semiautomatically fused to the CT data (Advantage Fusion 1.1.1; GE Medical Systems) using the nasion, top of the dens axis, and the internal occipital protuberance as landmarks. To locate the array within the midbrain, $1 \mathrm{~mm}$ slices of the fused images were compared with fixed human brain slices of similar dimension that were taken from the anatomical brain collection at the Department of Neuroanatomy in Hannover Medical University ${ }^{40}$. The alignment of the array and electrode sites with respect to the ICC anatomical layers, which hypothetically correspond to the frequency layers, was determined by comparing the fused images and fixed slices with previously published stained human midbrain sections ${ }^{34}$.
Implant fitting and testing. The implant processor separates the incoming sound into frequency bands through band-pass filtering. The envelope of each filtered signal is used to modulate the amplitude of an electrical pulse train presented to each site. For the subject, a 250 pps pulse train consisting of cathodic-leading biphasic pulses (100 $\mu$ s/phase, $45 \mu$ s inter-phase gap, monopolar configuration) was used. A pitch ordering across sites must be programmed into the implant processor to transmit the specific frequency components of the incoming sound to the correct sites during daily stimulation. The threshold (T) and upper comfortable (C) activation levels must also be programmed to limit the stimulation range for each site. For this study, the activation levels and pitch ordering over time were measured using the pulse parameters listed above. Sites 12-20 elicited non-auditory sensations and were inactivated. Details on the implant technology, fitting parameters, and testing procedures have been presented in previous publications ${ }^{39,41}$. Some of the earlier pitch data and activation levels presented in this paper were published previously ${ }^{39}$.

Activation levels. Each site was continuously stimulated for a duration of $500 \mathrm{~ms}$ repeated every $1 \mathrm{~s}$. The current level was first increased to elicit a moderate sound sensation and then decreased until the subject could no longer hear the stimulus, which was taken as $\mathrm{T}$ level. C level corresponded to a comfortable loudness obtained when increasing the current from $\mathrm{T}$ level. These measurements were repeated and levels were adjusted until consistent effects were obtained across trials. The loudness between two sites for all pairwise combinations was repeatedly compared to obtain a similar $\mathrm{C}$ sensation across all valid sites.

Pitch tests. Three different pitch tests were performed. Since site 7 was shorted to a distant non-auditory site and required higher activation levels (Fig. 3), it was excluded from analysis. Site 7 was still used for daily stimulation since it did not elicit any non-auditory sensations. Initially, a clear pitch ordering was not observed from these tests, and thus a site ordering for the processor was estimated based on qualitative responses from the subject during fitting $(0-4$ months in Fig. $4 \mathrm{~b})$. During the 4-month fitting session, the subject described sites 10 and 11 as having a "less comfortable" sensation. Those sites were inactivated and slight ordering adjustments were made for the $4-10$ month mapping based on qualitative responses from the subject. It was not until the 10 -month session and after additional pitch tests when it was realized that a systematic pitch ordering to AMI stimulation was present and consistent with the tonotopy observed in animals, and thus a tonotopic site ordering was implemented $(10-21$ months in Fig. $4 b)$.

Qualitative pitch scaling: Each site was stimulated with a $3 \mathrm{~s}$ pulse train at C level and the subject was asked to scale the perceived pitch from 0 to 5 (steps of 0.5 ) that most resembled the pitch level of familiar objects ( 0 : bass or boat horn, 2: man's voice, 3.5: woman's voice, 5 : bird chirping). These measurements were randomly repeated across sites for a total of $n$ times for each site ( $n$ values listed in Fig. 2 caption). For statistical analysis performed in Fig. 2a to compare the pitch values between sessions for each site, a two-tailed ranked unequal variance t-test was used as previously described $^{42}$. This method provides a conservative comparison without requiring normal distribution or equal variance assumptions. The Bonferroni correction was performed to compensate for the three comparisons for each site.

Quantitative pitch scaling: Each site was stimulated with a $500 \mathrm{~ms}$ pulse train repeated at $1 \mathrm{~Hz}$ for $3 \mathrm{~s}$ at $\mathrm{C}$ level. The subject was then asked to scale the pitch from 0 to 50 (integers) corresponding to the lowest and highest pitch, respectively. All sites were initially stimulated several times to familiarize the subject with the pitch range across sites. The measurements were then performed randomly across sites and repeated for a total of $n$ times for each site.

2-AFC pitch ranking: Two sites were sequentially stimulated with pulse trains at $C$ level each for $500 \mathrm{~ms}$ with an inter-stimulus gap of $500 \mathrm{~ms}$ and the subject was asked to select the stimulated site that elicited a higher pitch. All pairwise combinations were randomly stimulated $n$ times in which each pair was presented in each order $n / 2$ times (e.g. site 1 followed by site 2 versus site 2 followed by site 1 ). The total number of times one site had a higher pitch than another site was tabulated in an SxS matrix where S corresponded to the number of sites. For example, for 10 sites numbered 1 to 10 , the first column corresponded to site 1 while each row corresponded to site 1 through 10 . The total number of times site 1 elicited a higher pitch than site 2 was inserted into the first column-second row. The total number of times site 2 elicited a higher pitch than site 3 was inserted in the second column-third row, and so on. All diagonal values were set to zero. Then the sum along each column was calculated as the score for the corresponding site, and it was divided by the maximum score possible for each site. Since each site was compared with the remaining 9 sites $n$ times each (e.g., $n=10)$, the maximum score possible was 90 . The resultant ratio was multiplied by 100 (in percentage) in which 100 corresponds to the highest pitch.

1. Levelt, C. N. \& Hubener, M. Critical-period plasticity in the visual cortex. Annu Rev Neurosci 35, 309-330 (2012).

2. de Villers-Sidani, E. \& Merzenich, M. M. Lifelong plasticity in the rat auditory cortex: basic mechanisms and role of sensory experience. Prog Brain Res 191, 119-131 (2011).

3. Jones, E. G. Cortical and subcortical contributions to activity-dependent plasticity in primate somatosensory cortex. Annu Rev Neurosci 23, 1-37 (2000).

4. Fallon, J. B., Irvine, D. R. \& Shepherd, R. K. Neural prostheses and brain plasticity. J Neural Eng 6, 065008 (2009).

5. Keuroghlian, A. S. \& Knudsen, E. I. Adaptive auditory plasticity in developing and adult animals. Prog Neurobiol 82, 109-121 (2007). 
6. Buonomano, D. V. \& Merzenich, M. M. Cortical plasticity: from synapses to maps. Annu Rev Neurosci 21, 149-186 (1998).

7. Willott, J. F. \& Turner, J. G. Neural plasticity in the mouse inferior colliculus: relationship to hearing loss, augmented acoustic stimulation, and prepulse inhibition. Hear Res 147, 275-281 (2000).

8. Xiong, Y., Zhang, Y. \& Yan, J. The neurobiology of sound-specific auditory plasticity: a core neural circuit. Neurosci Biobehav Rev 33, 1178-1184 (2009).

9. Irvine, D. R., Rajan, R. \& McDermott, H. J. Injury-induced reorganization in adult auditory cortex and its perceptual consequences. Hear Res 147, 188-199 (2000).

10. Izquierdo, M. A., Gutierrez-Conde, P. M., Merchan, M. A. \& Malmierca, M. S Non-plastic reorganization of frequency coding in the inferior colliculus of the rat following noise-induced hearing loss. Neuroscience 154, 355-369 (2008).

11. Kamke, M. R., Brown, M. \& Irvine, D. R. Plasticity in the tonotopic organization of the medial geniculate body in adult cats following restricted unilateral cochlear lesions. J Comp Neurol 459, 355-367 (2003).

12. Fritz, J., Elhilali, M. \& Shamma, S. Active listening: task-dependent plasticity of spectrotemporal receptive fields in primary auditory cortex. Hear Res 206, 159-176 (2005).

13. Weinberger, N. M. \& Bakin, J. S. Learning-induced physiological memory in adult primary auditory cortex: receptive fields plasticity, model, and mechanisms. Audiol Neurootol 3, 145-167 (1998).

14. Suga, N. \& Ma, X. Multiparametric corticofugal modulation and plasticity in the auditory system. Nat Rev Neurosci 4, 783-794 (2003)

15. Kilgard, M. P. \& Merzenich, M. M. Cortical map reorganization enabled by nucleus basalis activity. Science 279, 1714-1718 (1998).

16. Engineer, N. D. et al. Reversing pathological neural activity using targeted plasticity. Nature 470, 101-104 (2011).

17. Guiraud, J. et al. Evidence of a tonotopic organization of the auditory cortex in cochlear implant users. J Neurosci 27, 7838-7846 (2007).

18. Fallon, J. B., Irvine, D. R. \& Shepherd, R. K. Cochlear implant use following neonatal deafness influences the cochleotopic organization of the primary auditory cortex in cats. J Comp Neurol 512, 101-114 (2009).

19. Irvine, D. R., Rajan, R. \& Smith, S. Effects of restricted cochlear lesions in adult cats on the frequency organization of the inferior colliculus. J Comp Neurol 467, 354-374 (2003).

20. Fallon, J. B., Irvine, D. R. \& Shepherd, R. K. Cochlear implants and brain plasticity. Hear Res 238, 110-117 (2008).

21. Lim, H. H. \& Anderson, D. J. Antidromic activation reveals tonotopically organized projections from primary auditory cortex to the central nucleus of the inferior colliculus in guinea pig. J Neurophysiol 97, 1413-1427 (2007).

22. Bajo, V. M., Nodal, F. R., Moore, D. R. \& King, A. J. The descending corticocollicular pathway mediates learning-induced auditory plasticity. Nat Neurosci 13, 253-260 (2010).

23. Merzenich, M. M. \& Reid, M. D. Representation of the cochlea within the inferior colliculus of the cat. Brain Res 77, 397-415 (1974).

24. Malmierca, M. S. et al. A discontinuous tonotopic organization in the inferior colliculus of the rat. J Neurosci 28, 4767-4776 (2008).

25. Schreiner, C. E. \& Langner, G. Laminar fine structure of frequency organization in auditory midbrain. Nature 388, 383-386 (1997).

26. Stiebler, I. \& Ehret, G. Inferior colliculus of the house mouse. I. A quantitative study of tonotopic organization, frequency representation, and tone-threshold distribution. J Comp Neurol 238, 65-76 (1985).

27. Lim, H. H. \& Anderson, D. J. Auditory cortical responses to electrical stimulation of the inferior colliculus: implications for an auditory midbrain implant. J Neurophysiol 96, 975-988 (2006).

28. Webster, W. R., Serviere, J., Crewther, D. \& Crewther, S. Iso-frequency 2-DG contours in the inferior colliculus of the awake monkey. Exp Brain Res $\mathbf{5 6}$ 425-437 (1984).

29. Harris, D. M., Shannon, R. V., Snyder, R. \& Carney, E. Multi-unit mapping of acoustic stimuli in gerbil inferior colliculus. Hear Res 108, 145-156 (1997).
30. Moore, D. R., Semple, M. N. \& Addison, P. D. Some acoustic properties of neurones in the ferret inferior colliculus. Brain Res 269, 69-82 (1983).

31. Harrison, R. V., Ibrahim, D. \& Mount, R. J. Plasticity of tonotopic maps in auditory midbrain following partial cochlear damage in the developing chinchilla. Exp Brain Res 123, 449-460 (1998).

32. Baumann, S. et al. Orthogonal representation of sound dimensions in the primate midbrain. Nat Neurosci 14, 423-425 (2011).

33. Oliver, D. L. in The Inferior Colliculus (eds J. A. Winer \& C. E. Schreiner) 69-114 (Springer Science + Business Media, Inc., 2005).

34. Geniec, P. \& Morest, D. K. The neuronal architecture of the human posterior colliculus. A study with the Golgi method. Acta Otolaryngol Suppl 295, 1-33 (1971).

35. Rajan, R. \& Irvine, D. R. Absence of plasticity of the frequency map in dorsal cochlear nucleus of adult cats after unilateral partial cochlear lesions. J Comp Neurol 399, 35-46 (1998).

36. Hofman, P. M., Van Riswick, J. G. \& Van Opstal, A. J. Relearning sound localization with new ears. Nat Neurosci 1, 417-421 (1998).

37. Knudsen, E. I. Instructed learning in the auditory localization pathway of the barn owl. Nature 417, 322-328 (2002).

38. Samii, A. et al. Auditory midbrain implant: a combined approach for vestibular schwannoma surgery and device implantation. Otol Neurotol 28, 31-38 (2007).

39. Lim, H. H. et al. Electrical stimulation of the midbrain for hearing restoration: insight into the functional organization of the human central auditory system. J Neurosci 27, 13541-13551 (2007).

40. Kretschmann, H. J. \& Weinrich, W. Cranial Neuroimaging and Clinical Neuroanatomy: Magnetic Resonance Imaging and Computed Tomography. 2nd edn (Thieme Medical Publishers, Inc., 1992).

41. Patrick, J. F., Busby, P. A. \& Gibson, P. J. The development of the Nucleus Freedom Cochlear implant system. Trends Amplif 10, 175-200 (2006).

42. Ruxton, G. D. The unequal variance t-test is an underused alternative to the Student's t-test and the Mann-Whitney U test. Behav Ecol 17, 688-690 (2006).

43. Lenarz, T., Lim, H. H., Reuter, G., Patrick, J. F. \& Lenarz, M. The auditory midbrain implant: a new auditory prosthesis for neural deafness-concept and device description. Otol Neurotol 27, 838-843 (2006).

\section{Acknowledgements}

We would like to thank the patient for her time and cooperation. We would also like to thank J. Patrick, J. Pesch, and F. Risi for their guidance and assistance in this research, and M. Braun, E. Lorentz, and S. Haumann for their contributions with the audiogram data. S. Offutt, M. Straka, C. Markovitz, B. Smith, C. Gloeckner, and M. Wojtczak provided helpful comments on the manuscript. This work was supported by Cochlear Limited, German Ministry of Education and Research 01GQ0816, and University of Minnesota start-up funds.

\section{Author contributions}

H.H.L., M.L. and T.L. designed the study. H.H.L., G.J. and M.L. collected the data. H.H.L. analyzed the data and prepared the manuscript with contributions from M.L., G.J. and T.L.

\section{Additional information}

Competing financial interests: The authors declare no competing financial interests.

License: This work is licensed under a Creative Commons

Attribution-NonCommercial-ShareAlike 3.0 Unported License. To view a copy of this license, visit http://creativecommons.org/licenses/by-nc-sa/3.0/

How to cite this article: Lim, H.H., Lenarz, M., Joseph, G. \& Lenarz, T. Frequency representation within the human brain: Stability versus plasticity. Sci. Rep. 3, 1474; DOI:10.1038/srep01474 (2013). 